\title{
A maximum density electrical insulating composite based on radiation-polymerized copolymer of isodecyl methacrylate and benzyl methacrylate with $\mathbf{W}$
}

\author{
Ilya Antonov ${ }^{1}$, Boris Tolochko ${ }^{1,2}$, Alexandr Varand $^{1,2}$, Mikhail Mikhailenko ${ }^{1,2}$, Ilya Eltsov $^{3}$, \\ Alexandr Bryazgin ${ }^{2}$, and Eugene Smirnov ${ }^{4}$ \\ ${ }^{1}$ Institute of Solid State Chemistry and Mechanochemistry SB RAS, Novosibirsk, 630128 Russian \\ Federation \\ ${ }^{2}$ Budker Institute of Nuclear Physics SB RAS, Novosibirsk, 630090 Russian Federation \\ ${ }^{3}$ Novosibirsk State University, Novosibirsk, 630090 Russian Federation \\ ${ }^{4}$ Russian Federal Nuclear Center - Zababakhin All-Russia Research Institute of Technical Physics, \\ Snezhinsk, 456770 Russian Federation
}

\begin{abstract}
Polymerization was carried out using the method of radiation initiation, for which benzyl methacrylate (BzMA) and isodecyl methacrylate (IDMA) were treated with a beam of accelerated electrons. It is shown that to obtain highly filled compositions, the most suitable method is the alternation of heating-cooling cycles with the gradual introduction of material particles. Tungsten composites were obtained with a calculated density in the range from 3 to $6.8 \mathrm{~g} / \mathrm{cm}^{3}$. The absence of percolation conductivity in coatings with tungsten was found. Peel adhesion of the composite coating to the surface was $1 \pm 0.3 \cdot 10^{-1} \mathrm{MPa}$ with volume fraction of tungsten more than $30 \%$.
\end{abstract}

\section{Introduction}

Composites based on poly(methyl methacrylate) (PMMA) and its derivatives have a number of desirable properties such as stability upon severe conditions, resistance to weathering and moisture, outstanding transparency and high surface resistivity. Due to these superior characteristics, PMMA has been widely used as sizer, sealer, optical fiber, binder, additive, coating and polishing agents, transparent neutron stopper, high voltage application, and outdoor electrical application [1]. However, traditional methods of polymerization are imperfect due to the necessity to remove the agent necessary for polymerization (initiator, inhibitor, emulsifier and so on) from polymers, which is a complex and costly procedure, and the residual agents in final products often deteriorate their optical and electronic properties. But the application of physical initiation agents allowed us to obtain HMW polymers (bulk, solution) without the use of initiators and inhibitors [2]. The resulting polymers make it possible to synthesize more filled composites in comparison with the composites based on high molecular weight polymers obtained by traditional methods. 
Though the polymers of the derivatives of ester methacrylic acid are not so widely used, they are to have a number of additional valuable properties. For example, poly(benzyl methacrylate)[3] reported to be more thermally stable than PMMA, as well as their copolymers and composites [4].

The aim of this work was to study the possibility of using radiation initiation for the polymerization(Fig. 1) of methacrylic acid esters with large substituents (isodecyl, benzyl), obtaining their copolymer and highly filled composites.
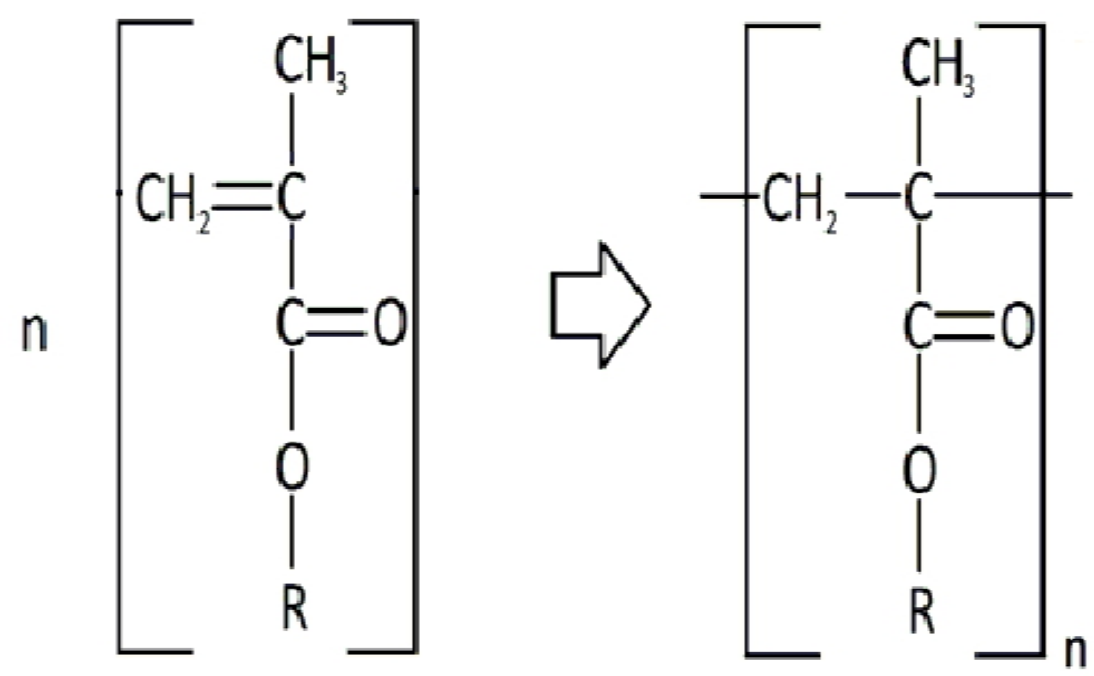

Fig. 1. Structural formula of copolymer $(\mathrm{R}=$-isodecyl and/or -benzyl).

\section{Experimental}

\subsection{Materials}

Ethyl acetate, ethanol, tetrahydrofuran stabilized, isodecyl methacrylate stabilized (Evonik), benzyl methacrylate stabilized (Evonik).

Tetrahydrofuran was purified before use by distillation under reduced pressure using a rotary evaporator. Purification of acrylates was carried out via separation funnel. A volume of distilled water was added to the same volume of acrylate, mixed, and after an hour water solution were separated from it. Bismuth oxide nanoparticles were obtained by electron beam evaporation [5]. Ultrafine tungsten was obtained by electric explosion of a conductor in an argon atmosphere and packed in glass ampoules under inert atmosphere.

\subsection{Synthesis of copolymer I1-B1}

Polymerization was carried out using the method of radiation initiation, for which benzyl methacrylate (BzMA) and isodecyl methacrylate (IDMA) were treated with a beam of accelerated electrons from the ILU-6 accelerator (BINP SB RAS) with electron energy of $2.4 \mathrm{MeV}$, pulse beam current of $320 \mathrm{~mA}$, pulse duration of $0.6 \mathrm{~ms}$, pulse repetition rate of $2.5 \mathrm{~Hz}$, underbeam transportation velocity of $2 \mathrm{~cm} / \mathrm{s}$. Dose is $20 \mathrm{kGy}(\mathrm{J} / \mathrm{g})$. 
The homogeneity of the copolymer I1-B1 prepared at a temperature of $70{ }^{\circ} \mathrm{C}$ (processing time $\sim 24$ hours) was checked by separating fractions by fractional precipitation from the ethyl acetate solution by adding ethanol.

\subsection{Characterization}

Thermal analysis was conducted on Calorimeter DSC-200 F3 MAIA (Netzsch, Germany) with aluminium crucibles, heating rate $10{ }^{\circ} \mathrm{C} / \mathrm{min}$, temperature range from 0 to $250{ }^{\circ} \mathrm{C}$ under argon atmosphere.

Electron microscopy studies were carried out with a scanning electron microscope SN 3400 (Hitachi, Japan) with XRF analyzer INCA (Oxford Instruments, UK).

To study the electrophysical properties of the coating, a suspension was applied to a copper plate so that the thickness of the coating was about $50 \mu \mathrm{m}$. Electrodes were attached to the copper plate: one electrode to the brushed copper surface, the other to the applied coating. Voltage was applied using a Keithley 248 power supply, and current was measured using a multimeter Keysight 34461 A. Main measurements were taken with a Megohmmeter F 4102 / 2-1M (Russia).

Adhesion was determined with an adhesion tester «Konstanta AC» (ISO 4624). Than dolly was glued to the composite on the copper plate with epoxide glue. Measurements were taken 24 hours after glue was applied. Measurements were taken 10 times.

\section{Results and discussion}

Copolymer solutions have an anomalous dependence of viscosity on temperature - an increase in viscosity is observed with temperature rise, which is characteristic of the coilchain transition [6]. This property was used to obtain composites in solutions. The heating and cooling cycles were alternated with the gradual addition of the particles.

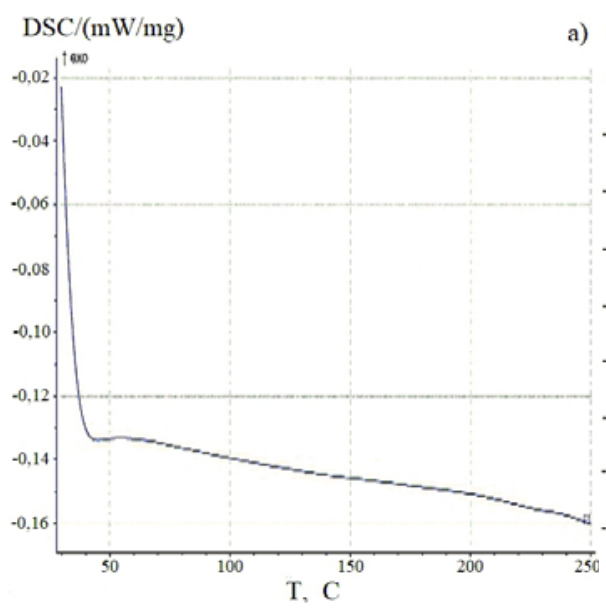

a) $\mathrm{DSC} /(\mathrm{mW} / \mathrm{mg})$

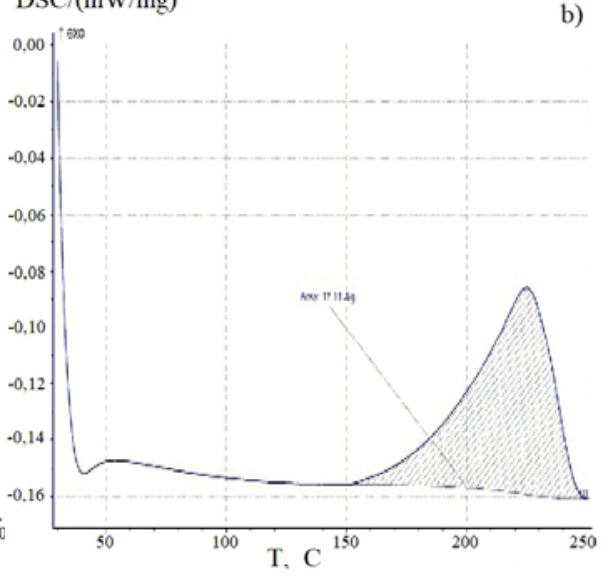

Fig. 2. DSC of the composite with tungsten a) and with bismuth oxide b).

Tungsten particles, obtained by means of electric explosion and stabilized with stearic acid and agglomerated, were used as a filler for the preparation of composites. The obtained tungsten particles were fractionated. To obtain composites, an average fraction of 20-40 $\mu \mathrm{m}$ was used, the size of individual particles in which did not exceed $5 \mu \mathrm{m}$. 
Due to the absence of direct contact of tungsten particles with air, the composites contain no oxide or its fraction is extremely insignificant. Figure 2 shows the results of a DSC study of the compositions of tungsten and bismuth oxide (shown for comparison). In the case of the composite with bismuth oxide, at a temperature above the temperature of the plastic flow of the polymer, an exothermic effect is observed, associated with the reduction of the oxide by the copolymer. In the case of the composite with tungsten, this effect is absent. A number of composites with a density ranging from 3 to $6.8 \mathrm{~g} / \mathrm{cm}^{3}$ have been obtained.

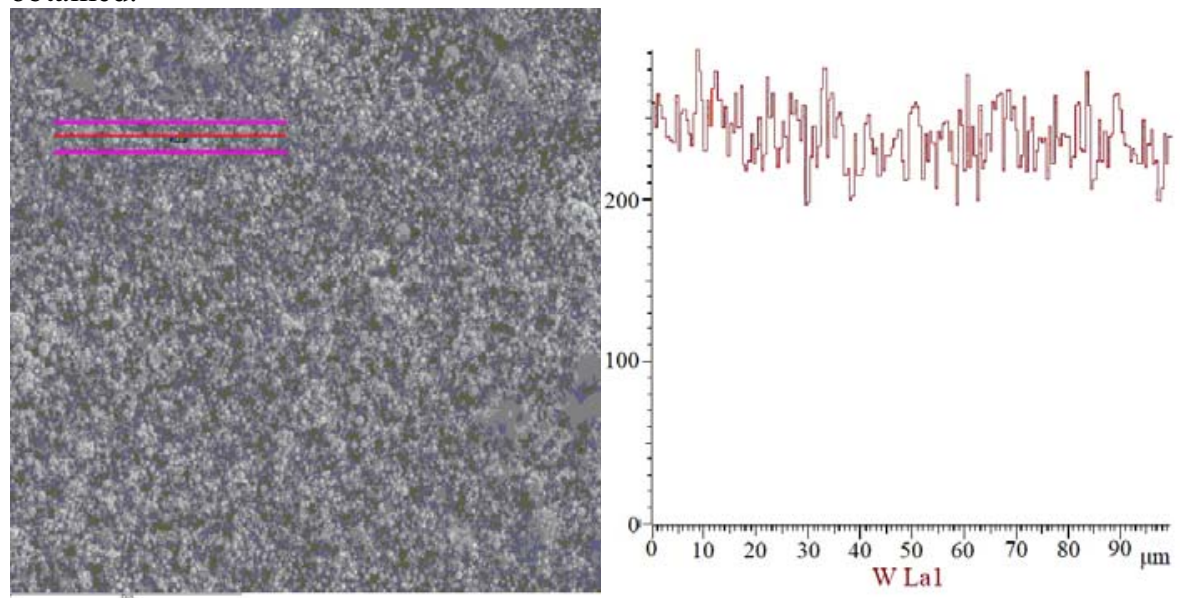

Fig. 3. Distribution of tungsten for samples with the volume fraction of filler over $30 \%$.

The resulting compositions were applied to copper plates. The results of scanning the obtained coatings to determine elemental composition using an energy-dispersive analyzer of the electron microscope indicate an uniform distribution of tungsten for samples with a volume fraction of the filler of more than $30 \%$ (Fig. 3).

The electrophysical properties of the coating were also investigated. Electrophysical studies have shown the absence of percolation conductivity in the coating with a calculated density of $6.8 \mathrm{~g} / \mathrm{cm}^{3}$. The results are shown in Table 1 .

Table 1. Electrophysical properties of the coating.

\begin{tabular}{|c|c|c|c|}
\hline $\mathrm{N}$ & $\begin{array}{c}\text { Number } \\
\text { of layers }\end{array}$ & Resistance at $1000 \mathrm{~V}, \mathrm{k} \Omega$ & Resistance at $2500 \mathrm{~V}, \mathrm{k} \Omega$ \\
\hline 1 & 1 & 50 & breakdown \\
\hline 2 & 2 & $250-300$ & 25 \\
\hline 3 & 3 & $750-800$ & $250-300$ \\
\hline
\end{tabular}

Adhesion was determined to be $1 \pm 0.3 \cdot 10^{-1} \mathrm{MPa}$ for the composites with a volume tungsten fraction of more than $30 \%$. Measurement error was calculated as standard deviation after ten measurements. 


\section{Summary}

It is shown that the most suitable method to obtain highly filled compositions is the alternation of heating and cooling polymer solution with the gradual introduction of material particles. Tungsten composites were obtained with a calculated density in the range from 3 to $6.8 \mathrm{~g} / \mathrm{cm}^{3}$. The absence of percolation conductivity in coatings with tungsten was found. Peel adhesion of the composite coating(with the volume fraction of tungsten more than $30 \%$ ) to the surface was $1 \pm 0.3 \cdot 10^{-1} \mathrm{MPa}$.

This work was supported by the Ministry of Education and Science of the Russian Federation (project No. AAAA-A19-119062690004-0).

\section{References}

1. W.S. Lyoo, S.K. Noh, J.H. Yeum, G.C. Kang, H.D. Ghim, J. Lee, B.C. Ji, Fibers Polym. 5, 75 (2004)

2. M. Yuan, L. Xu, X. Cui, J. Lv, P. Zhang. H. Tang, Polymers 12, 2747 (2020)

3. T.G. Obertia, M.M. Schiavoni, M.S. Cortizo, Rad. Phys. Chem., 77, 597 (2008)

4. N. Ayaz, F. Bezgin, K. Demirell, ISRN Pol. Sci. 352759 (2012)

5. B.P. Tolochko, E.I. Antokhin, Yu.M. Yukhin, V.S. Eliseev, M.G. Golkovsky, N.Z. Lyakhov, RF Patent No. 2426625 (2011)

6. T.M. Birshtein, V.A. Pryamitsyn, Macromolecules 24, 1554 (1991) 